University of Nebraska - Lincoln

DigitalCommons@University of Nebraska - Lincoln

2011

Estimating age from recapture data: integrating incremental growth measures with ancillary data to infer age-at-length

Mitchell J. Eaton

William A. Link

Follow this and additional works at: https://digitalcommons.unl.edu/usgsstaffpub

Part of the Geology Commons, Oceanography and Atmospheric Sciences and Meteorology Commons, Other Earth Sciences Commons, and the Other Environmental Sciences Commons

This Article is brought to you for free and open access by the US Geological Survey at DigitalCommons@University of Nebraska - Lincoln. It has been accepted for inclusion in USGS Staff -- Published Research by an authorized administrator of DigitalCommons@University of Nebraska - Lincoln. 


\title{
Estimating age from recapture data: integrating incremental growth measures with ancillary data to infer age-at-length
}

\author{
Mitchell J. EAton ${ }^{1}$ And William A. Link \\ USGS Patuxent Wildlife Research Center, 12100 Beech Forest Rd., Laurel, Maryland 20708 USA
}

\begin{abstract}
Estimating the age of individuals in wild populations can be of fundamental importance for answering ecological questions, modeling population demographics, and managing exploited or threatened species. Significant effort has been devoted to determining age through the use of growth annuli, secondary physical characteristics related to age, and growth models. Many species, however, either do not exhibit physical characteristics useful for independent age validation or are too rare to justify sacrificing a large number of individuals to establish the relationship between size and age. Length-at-age models are well represented in the fisheries and other wildlife management literature. Many of these models overlook variation in growth rates of individuals and consider growth parameters as population parameters. More recent models have taken advantage of hierarchical structuring of parameters and Bayesian inference methods to allow for variation among individuals as functions of environmental covariates or individual-specific random effects. Here, we describe hierarchical models in which growth curves vary as individual-specific stochastic processes, and we show how these models can be fit using capture-recapture data for animals of unknown age along with data for animals of known age. We combine these independent data sources in a Bayesian analysis, distinguishing natural variation (among and within individuals) from measurement error. We illustrate using data for African dwarf crocodiles, comparing von Bertalanffy and logistic growth models. The analysis provides the means of predicting crocodile age, given a single measurement of head length. The von Bertalanffy was much better supported than the logistic growth model and predicted that dwarf crocodiles grow from $19.4 \mathrm{~cm}$ total length at birth to $32.9 \mathrm{~cm}$ in the first year and $45.3 \mathrm{~cm}$ by the end of their second year. Based on the minimum size of females observed with hatchlings, reproductive maturity was estimated to be at nine years. These size benchmarks are believed to represent thresholds for important demographic parameters; improved estimates of age, therefore, will increase the precision of population projection models. The modeling approach that we present can be applied to other species and offers significant advantages when multiple sources of data are available and traditional aging techniques are not practical.
\end{abstract}

Key words: African dwarf crocodile; age-at-length; Bayesian analysis; Gamma process; growth model; hierarchical models; mark-recapture; MCMC, Markov chain Monte Carlo; model selection; Osteolaemus tetraspis; posterior predictive distribution; random effects.

\section{INTRODUCTION}

Aging is fundamental to the evolution and ecology of all species. Age-specific declines in selective pressures and somatic growth result in decreased reproductive and survival rates, which, in turn, are primary determinants of population growth (Cole 1954, Stearns 1992). Thus, many population dynamics models require knowledge of the ages of individuals to relate distinct demographic rates to age class (Williams et al. 2002). Even if stagestructured models are used to correlate demographic processes to classes (size or stage) rather than age, predicting transition rates between these classes relies on estimates of age-dependent growth over time (Caswell

Manuscript received 25 March 2010; revised 23 February 2011; accepted 3 March 2011. Corresponding Editor: E. M. Schauber.

${ }^{1}$ E-mail: meaton@usgs.gov
2001). Age estimation has also been used when testing basic ecological and evolutionary hypotheses concerning growth patterns (e.g., Bjorndal et al. 2003) and the influence of environment, species interactions, or resources on reproductive behavior (e.g., Ims 1990, Rachlow and Bowyer 1991, Franken and Hik 2004). Imprecise age estimates can reduce effectiveness in managing endangered or exploited species by biasing demographic parameters and extinction probabilities (Leopold 1933, Alexander 1958, Cailliet et al. 1992).

Knowing the precise age of a free-living animal is rare, requiring that individuals or cohorts be tagged as newborns and followed until death. When obtaining complete individual histories is not feasible, age must instead be estimated. Such estimates are often based on counts of growth annuli deposited in teeth, otoliths, scales, bones, and other hard tissue. These methods may be inappropriate because growth annuli are absent, or unreliable due to bone lamina resorption or irregular 
deposition patterns (Hutton 1987a, Spencer 2002, Avens et al. 2009). Additional aging techniques include tooth eruption and wear patterns in ungulates and carnivores (Wilson et al. 1984, Clarke et al. 1992, Clawson and Causey 1995, Moore et al. 1995, Stander 1997), eye-lens weight in mammals (Hearn and Mercer 1988), closure of the epiphyseal plate in long bones (Kohn et al. 1997), and a controversial technique based on chromosomal telomere length (Haussmann and Vleck 2002). Several of these methods require that animals be sacrificed, a constraint that is not justifiable for many species, given the large sample sizes needed. Others techniques may be too imprecise to be useful for the particular study question. In such cases, the alternative to direct age estimation is the development of isometric or allometric growth curves to relate age to measurements of body size, proportion, or mass (Cheng and Kuk 2002, Laundré and Hernández 2002, Reilly 2002, Liu et al. 2009).

The development of growth models for individuals in wild populations has benefited from a long history in animal ecology. This development is most apparent in fisheries research, where length data are routinely collected by commercial fisheries and growth models are tested using subsamples of independent measures of age (e.g., otoliths). Traditionally, growth model parameters have been considered as average population parameters and are estimated using nonlinear regression methods from size or length-at-age data where interindividual variability is ignored (Kimura 1980, Wilson et al. 1984, Cailliet et al. 1992). Marine and freshwater populations have also served as model systems for more sophisticated approaches to estimating growth. Advances in growth modeling include the explicit recognition of individual heterogeneity in growth parameters via the addition of random-effects terms (e.g., James 1991, Pilling et al. 2002, Tovar-Avila et al. 2009). Other studies have evaluated the impact of error or uncertainty in age determination on estimates of growth (Leberg et al. 1989), including incorporation of process and measurement error as a random effect in hierarchical models (Schwarz and Runge 2009). Growth has been modeled as a function of sex, geographic location, year class, environmental factors, or other covariates using nonlinear fixed-effects models (i.e., Kimura 2008), linear mixed-effects models (Hart and Chute 2009), or metaanalyses of multiple fisheries stocks using mixed-effects hierarchical models (Helser and Lai 2004, Helser et al. 2007). Bayesian and hierarchical Bayesian modeling approaches have been used widely in fisheries and terrestrial science, including applications for estimating individual growth (Pilling et al. 2002, He and Bence 2007, Helser et al. 2007) and inference on population demographic parameters (Wade 2000, Gross et al. 2002, Moore and Read 2008). The analysis of capturerecapture data (Bacon-Shone 1988, Clark et al. 2005) and the integration of disparate data sets (Wade 2000, Clark 2005, Moore and Read 2008) have also benefited from advances in Bayesian modeling.
Here, we describe hierarchical models in which growth curves vary as individual-specific stochastic processes. Some model parameters can be estimated using capture-recapture data for animals of unknown age; others require data for animals of known age. We combine independent data sources in a Bayesian analysis, distinguishing natural variation (among and within individuals) from measurement error. Our purpose in this modeling exercise is to produce predictions of age from measurements of size, with interval predictions appropriately describing uncertainties due to variation among animals, measurement error, and sampling variation in our data.

Our approach facilitates inclusion of relevant covariates, prior knowledge about parameters, and ancillary data into a single, integrated framework to then predict the age of animals captured at a single occasion. In the example that we present, supplemental data consist of measurements of young animals captured once and modeled to estimate birth size, and a limited number of measurements from known-age individuals (i.e., newborns, age $=0$ ). Bayesian analysis, implemented using Markov chain Monte Carlo, provides a formal basis for multimodel inference, which we use for comparing alternative growth models (Link and Barker 2010). Although our use of hierarchical Bayesian inference for modeling individual growth is not novel, the integration of capture-recapture data with auxiliary information should be a valuable addition to growth models used to estimate age-at-size, especially for populations at low abundance or for difficult-to-study species (Webb et al. 1983, Hutton 1987a).

We apply our model to growth increment data collected during a four-year capture-recapture study on the Central African dwarf crocodile (Osteolaemus tetraspis, Cope). Traditional aging methods have been problematic when applied to crocodilians due to their longevity, slow and variable body growth (attributed to varying environmental conditions and ectothermic metabolism), and lack of morphological features relating to age. Advances in skeletochronology are promising (e.g., Tucker 1997, Rasch et al. 2000), but resorption and remodeling of bone in older animals and in reproductive females limit the accuracy and usefulness of this technique in crocodiles and other reptiles (Hutton 1987b, Tucker 1997, Bjorndal et al. 1998). Skeletochronology, with the exception of analyzing lamina in crocodilian osteoderms (Hutton 1987b), also requires animals to be sacrificed. The dwarf crocodile is endemic to closed-canopy forests of Central and West Africa and is the smallest of the world's crocodilians (see Plate 1). The species is difficult to study in the wild, due to its nocturnal behavior and preference for dense vegetation cover, and is threatened with overhunting for the bushmeat trade (Waitkuwait 1989, Kofron 1992, Riley and Huchzer-meyer 1999, Thorbjarnarson and Eaton 2004, Eaton et al. 2009). Very little is known of the ecology, life history, or demography of the dwarf 
crocodile. Progress toward reliable estimates of age, including age at reproductive maturity and other transitional demographic stages, will improve population models and our ability to manage this and other intensively exploited or threatened species (Hutton 1987a).

\section{Methods \\ Study site}

Field studies were conducted on the central coast of the Republic of Gabon (Loango National Park, approximately $2^{\circ} 18^{\prime} \mathrm{S}$, 9 $36^{\prime} \mathrm{E}$; (Eaton 2006). The national park is located within the Ogooué River watershed and is characterized by an edaphic paleodune vegetation community and gallery mosaic forests interspersed with savannas. The region contains a diversity of interior freshwater and coastal habitat, including swamp forests, seasonally inundated upland river forests, and networks of brackish lagoons and associated waterways.

\section{Data collection}

Surveys were conducted between July and November each year from 2004 to 2007, with most observations made in July and August. Surveys took place at night, on foot or in canoe, with one spotter using a headlamp or flashlight to locate and approach animals and one to two assistants helping with captures and measuring. Crocodiles were captured by snare pole, tongs, or by hand to ensure representation of all size classes. Length measurements were recorded using a flexible steel tape and included head length (HL, measured from the tip of the snout to the medial posterior edge of the supraoccipital plate), snout-vent length (SVL, measured ventrally to the posterior termination of the cloaca), and total length (TL, measured ventrally to the tip of tail, noting whether the tail was complete or damaged). Body mass was recorded with a spring scale $(500 \mathrm{~g}$ to $20 \mathrm{~kg}$; Pesola AG, Baar, Switzerland) selected based on the size of the crocodile. Sex was determined by probing the cloaca and visually examining the genitalia. Crocodiles were individually marked by notching a combination of caudal scutes, and then were released at the point of capture. All surveyed streams and lagoons were revisited at least twice per season and during each year of the study. Measurements recorded for crocodiles captured on more than one occasion constitute the basis of the growth analysis and, hence, are described as our primary data set (see Appendix A). The primary data can be used to estimate all parameters of the growth models that we consider, except one parameter related to birth size.

To provide information necessary to fully parameterize our growth models, we included a supplementary data set (Appendix B) containing the measurements of 47 of the smallest dwarf crocodile hatchlings captured during the study (none of which was recaptured) and four captive hatchling dwarf crocodiles. These ancillary data were used to develop a model-based prediction of birth size. Six of the 51 young crocodiles were of known age (0-27 days), including two wild dwarf crocodiles caught emerging from a nest (i.e., age zero) and the four captive animals [two from Lincoln Park Zoo (Chicago, Illinois, USA; D. Bohem, personal communication) and two from Toronto Zoo (Toronto, Canada; A. Lentini, personal communication)]. Of these six known-age animals, four had measurements of TL only, one had only a HL measurement, and one had both TL and HL measures. The remaining 45 hatchlings were of unknown age and hence were not used to inform birth size directly, but only the relationship between HL and TL for small individuals.

\section{Growth models}

The primary data set included recapture measurements of 46 crocodiles. A total of 97 head length measurements were obtained from 41 animals captured twice and 5 captured three times. The majority (65\%) of captures occurred in July and August. The interval between captures was $544 \pm 293.8$ days (mean \pm SD; minimum 40 days, maximum 1208 days; Appendix A: Table A1). The data included animals ranging in size (first capture) from 30 to $153 \mathrm{~cm} \mathrm{TL}$, encompassing nearly the full span of body sizes observed in the wild. We chose to model head length because of its strong isometric relationship to total length, biases introduced in TL measurements by tail-tip amputations, and lower error associated with bone to bone measurements (Webb et al. 1983). We considered two common models relating body size to age. These were the von Bertalanffy (VB) growth model (Fabens 1965), where an individual of age $A$ has size

$$
S(A)=a\left(1-b e^{-k A}\right)
$$

and the logistic growth (LG) model (Schoener and Schoener 1978), where

$$
S(A)=\frac{a(1-b) e^{k A}}{b+(1-b) e^{k A}} .
$$

We have parameterized the models so that $a$ is the asymptotic head length $(S(\infty)=a), b$ relates birth size to asymptotic size $(S(0)=a(1-b))$, and $k>0$ is a growth rate coefficient.

\section{Individual-specific variation}

One would not anticipate that all individuals will have the same asymptotic size, nor that individual growth patterns will conform exactly to the pattern $S(A)$ described by Eqs. 1 or 2. We thus describe a model under which the true head size for animal $i$ at age $A$, denoted $H_{i}(A)$, is a single realization of a nondecreasing stochastic process, with $S(A)$ being the population mean for individuals of age $A$.

Our model involves Gamma processes. A Gamma process is a type of Lévy process, these latter being examples of continuous-time Markov processes (for 


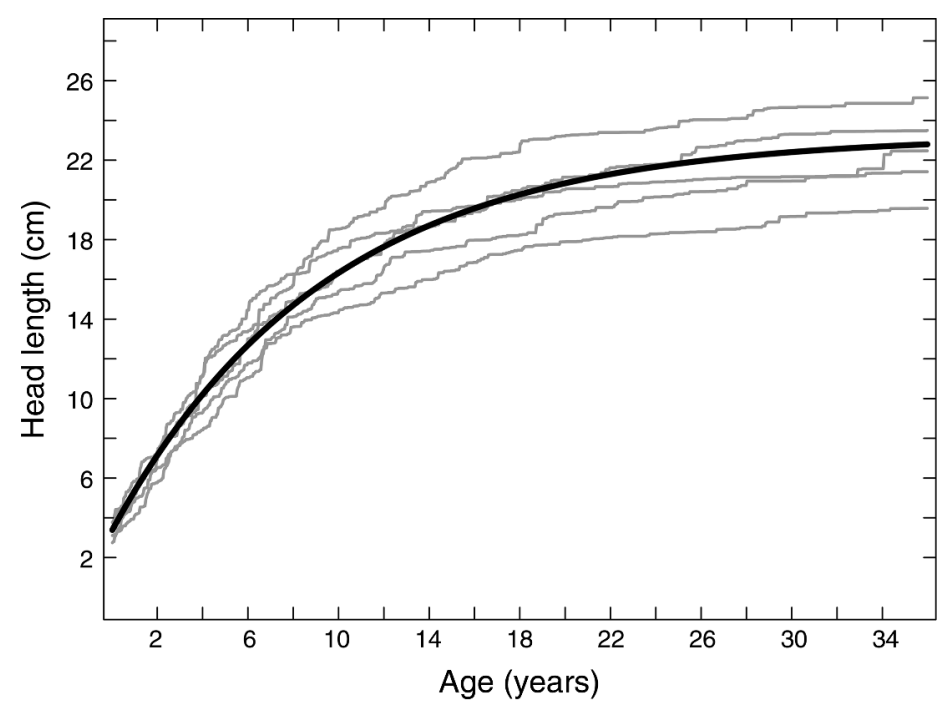

FIG. 1. Five simulated growth curves for African dwarf crocodiles (Osteolaemus tetraspis) under a von Bertalanffy Gamma process model (gray lines), showing variation within and among individuals. The black curve is the population expected value under the same model. Parameter values used are the median point estimates derived from the recapture analysis, $a=23.2, b$ $=0.86, k=0.29$, and $\lambda=6.6$, where $a$ is asymptotic head length, $b$ is a constant relating birth size to asymptotic size, $k$ is a growth rate coefficient (scaled to 1000 -day intervals), and $\lambda$ is the ratio of mean to variance for describing individual heterogeneity. detailed mathematical exposition, see Applebaum [2009]; for applications to growth models, see Russo et al. [2009]). For our purposes it suffices to note three features of a Gamma process $\eta(x)$ with parameters $p, \lambda$ $>0$, where $\eta(x)$ is indexed by the positive real numbers $x>0$ :

1) $\eta(0)=0$. For $x>0, \eta(x) \sim \Gamma(p x, \lambda)$, i.e., $\eta(x)$ has a Gamma distribution with mean $p x / \lambda$ and variance $p x /$ $\lambda^{2}$.

2) The increment $\eta\left(x_{2}\right)-\eta\left(x_{1}\right)$ over the interval $\left(x_{1}\right.$, $\left.x_{2}\right]$ is also a Gamma random variable, $\left[\eta\left(x_{2}\right)-\eta\left(x_{1}\right)\right] \sim$ $\Gamma\left(p\left(x_{2}-x_{1}\right), \lambda\right)$, where $x_{1}<x_{2}$.

3) Increments for disjoint intervals are independent.

We model individual growth as $H_{i}(A)=\eta_{i}(S(A))$, where $S(A)$ is given by Eqs. 1 or 2 ; here $\eta_{i}(x)$ are independent and identically distributed Gamma processes with $p=\lambda$. This model specifies that an individual's true head size is a nondecreasing stochastic process, and that for individuals of age $A$, the population mean and variance are $S(A)$ and $S(A) / \lambda$, respectively. Fig. 1 displays growth curves for five individuals, simulated under the von Bertalanffy model, with $a=23.2, b=0.86, k=0.29$, and $\lambda=6.6$, along with the population mean curve.

Suppose animal $i$ is captured on $m>1$ sampling occasions, at unknown ages

$$
A_{i 1}<A_{i 2}<\cdots<A_{\text {im }} .
$$

Because the VB and LG curves are strictly increasing,

$$
0<S\left(A_{i 1}\right)<S\left(A_{i 2}\right)<\cdots<S\left(A_{i m}\right) .
$$

That is, the expected head sizes partition the interval $(0$, $S\left(A_{\text {im }}\right)$ ] into $m$ disjoint intervals

$$
\begin{array}{r}
\left(0, S\left(A_{i 1}\right)\right],\left(S\left(A_{i 1}\right), S\left(A_{i 2}\right)\right],\left(S\left(A_{i 2}\right), S\left(A_{i 3}\right)\right] \\
\ldots,\left(S\left(A_{i m-1}\right), S\left(A_{i m}\right)\right] .
\end{array}
$$

Changes in the Gamma process $\eta_{i}(x)$ over these $m$ intervals are thus independent Gamma random variables. These changes are $I_{i 1}=\eta_{i}\left(S\left(A_{i 1}\right)\right)-\eta_{i}(0)=H_{i}\left(A_{i 1}\right)$ -0 , the true head size at first capture, and $I_{i j}=\eta_{i}\left(S\left(A_{i j}\right)\right)$ - $\eta_{i}\left(S\left(A_{i, j-1}\right)\right)=H_{i}\left(A_{i j}\right)-H_{i}\left(A_{i, j-1}\right)$, the change in true head size over interval $j$, for $j=2,3, \ldots, m$.

Our model thus describes the true head sizes on the $m$ sampling occasions as sums of independent Gamma random variables, $H_{i}\left(A_{i j}\right)=\Sigma_{k=1}^{j} I_{i k}$. Letting $I_{i j}^{*}=S\left(A_{i, j}\right)-$ $S\left(A_{i, j-1}\right)$, we have $I_{i j} \sim \Gamma\left(\lambda I_{i j}^{*}, \lambda\right)$; the mean of $I_{i j}$ is $I_{i j}^{*}$, and the variance is $I_{i j}^{*} / \lambda$.

Under the VB model, if $j>1$,

$$
S\left(A_{i j}\right)=S\left(A_{i, j-1}\right)+\left(a-S\left(A_{i, j-1}\right)\right)\left(1-e^{-k \Delta i j}\right)
$$

where $\Delta_{i j}=A_{i j}-A_{i, j-1}$, the time between capture occasions, is known. Thus given $a, k$, the expected head size at the (unknown) age of first capture $S\left(A_{i 1}\right)$, and the times between captures $\Delta_{i j}$, one can compute all of the expected head sizes $S\left(A_{i j}\right)$ through recursive application of Eq. 3, and from these the values $I_{i j}^{*}=S\left(A_{i j}\right)-S\left(A_{i, j-1}\right)$ needed to describe the distributions of the Gamma increments $I_{i j}$. Our model for true head size thus includes one individual-specific parameter, $S\left(A_{i 1}\right)$, and three parameters common to the population $(a, k$, and $\lambda)$. Similarly, under the LG model:

$$
\frac{1}{S\left(A_{i j}\right)}=\frac{1}{S\left(A_{i, j-1}\right)}+\left(\frac{1}{a}-\frac{1}{S\left(A_{i, j-1}\right)}\right)\left(1-e^{-k \Delta i j}\right) .
$$

Once again, our model for true head size is seen to be fully parameterized by values $S\left(A_{i 1}\right), a, k$, and $\lambda$.

\section{Bayesian analysis of recapture data}

We model head length measurement $h_{i j}$ for individual $i$ at capture occasion $j$ in our primary data set as

$$
h_{i j}=H_{i}\left(A_{i j}\right)+\varepsilon_{i j}^{\mathrm{R}}
$$

where $\varepsilon_{i j}^{\mathrm{R}}$ are independent, normally distributed mea- 


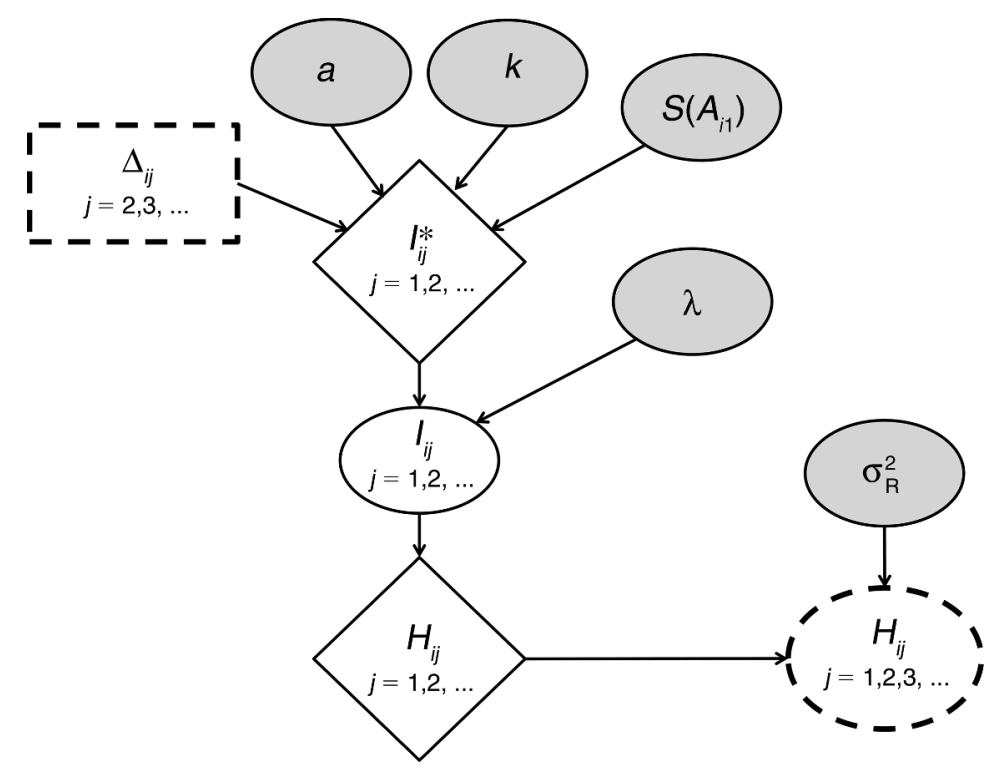

FIG. 2. Directed acyclical graph describing the recapture model. Subscripts $i$ and $j$ index individual and capture occasion, respectively; $\Delta$ is the time between capture occasions, $S(A)$ is the expected head length of an animal of age $A$, $I_{i j}$ is the change in true head length of individual $i$ over the capture interval from $j-1$ to $j, I_{i j}^{*}$ is the expected value of $I_{i j}, H$ refers to the true head length of an individual, $h$ is the measured head length, and $\sigma_{\mathrm{R}}^{2}$ is the measurement error variance. See Methods: Individual-specific variation and Bayesian analysis of recapture data for details of parameters included in the recapture model.

surement errors, having mean zero and common variance $\sigma_{R}^{2}$. The super- and subscripts $R$ (recapture) in the error and variance terms are included to distinguish these from other error terms to be described.

A directed acyclic graph (Fig. 2) indicates the hierarchical model structure underlying head length measurements. Observed quantities are indicated by nodes outlined with dashed lines. Stochastic elements of the model are indicated by ovals; unshaded ovals have distributions determined by the nodes with arrows leading to them and shaded ovals indicate model parameters requiring priors for Bayesian analysis. Derived quantities are indicated by tilted rectangles; these are functions of the nodes with arrows leading to them.

We assigned uniform priors on $[0,100]$ to $S\left(A_{i 1}\right)$, the expected size of an animal at the unknown age of first capture for animal $i$, and diffuse mean zero normal priors $(\mathrm{SD}=1000)$ to the logarithms of $a$ and $k$; we denote this prior by $N\left(0,1000^{2}\right)$. The measurement error variance $\sigma_{\mathrm{R}}^{2}$ was assigned a diffuse inverse gamma distribution (scale $=$ shape $=0.001)$, denoted $\mathrm{IG}(0.001,0.001)$.

We considered models in which parameters $a$ and $k$ were allowed to vary by sex to test for gender-specific differences in growth or asymptotic size. We compared the VB and LG models using Bayesian multimodel inference based on reversible jump Markov chain Monte Carlo, MCMC (Link and Barker 2010).

\section{Modeling supplementary data}

The primary data set (Appendix A; head length measurements for crocodiles of unknown age) provides no information about the parameter $b$; the parameter is not involved in calculating expected increments $I_{i j}^{*}$ using Eqs. 3 or 4 . Given that parameter $b$ relates birth size to asymptotic size $a$, it is not surprising that we cannot estimate it using data from animals of unknown age; parameter $b$ was estimated using a supplementary data set, as we now describe.

The supplemental data set (Appendix B) consisted of data for 51 young crocodiles and included a combination of TL and HL measurements:

$$
\mathrm{HL}_{i}=\mu_{i}^{\mathrm{HL}}+\varepsilon_{i}^{\mathrm{HL}}
$$

where $\mu_{i}^{\mathrm{HL}}=\alpha_{\mathrm{HL}}+\beta_{\mathrm{HL}}$ Age $_{i}$, and

$$
\mathrm{TL}_{i}=\mu_{i}^{\mathrm{TL}}+\varepsilon_{i}^{\mathrm{TL}}
$$

where $\mathrm{TL}_{i}$ is the true total length of individual $i$ and

$$
\mu_{i}^{\mathrm{TL}}=\alpha_{\mathrm{TL}}+\beta_{\mathrm{TL}} \mu_{i}^{\mathrm{HL}} .
$$

Our choice of a linear growth model for hatchlings was motivated by a desire for reliable, model-insensitive predictions of birth size; we note that the choice is consistent with either the VB or the LG model, both of which are nearly linear over the size range of young crocodiles. Regression coefficients $\alpha$ and $\beta$ were assigned $N\left(0,100^{2}\right)$ priors. Errors $\varepsilon_{i}^{\mathrm{HL}}$ and $\varepsilon_{i}^{\mathrm{TL}}$ were assumed to be normal random variables with a mean of zero and variances $\sigma_{\mathrm{HL}}^{2}$ and $\sigma_{\mathrm{TL}}^{2}$, respectively; these were assigned $\mathrm{IG}(0.001,0.001)$ priors. For the 45 smallest individuals of unknown age, we assigned uniform priors for Age over the range 0-100 days. Regression estimates of 


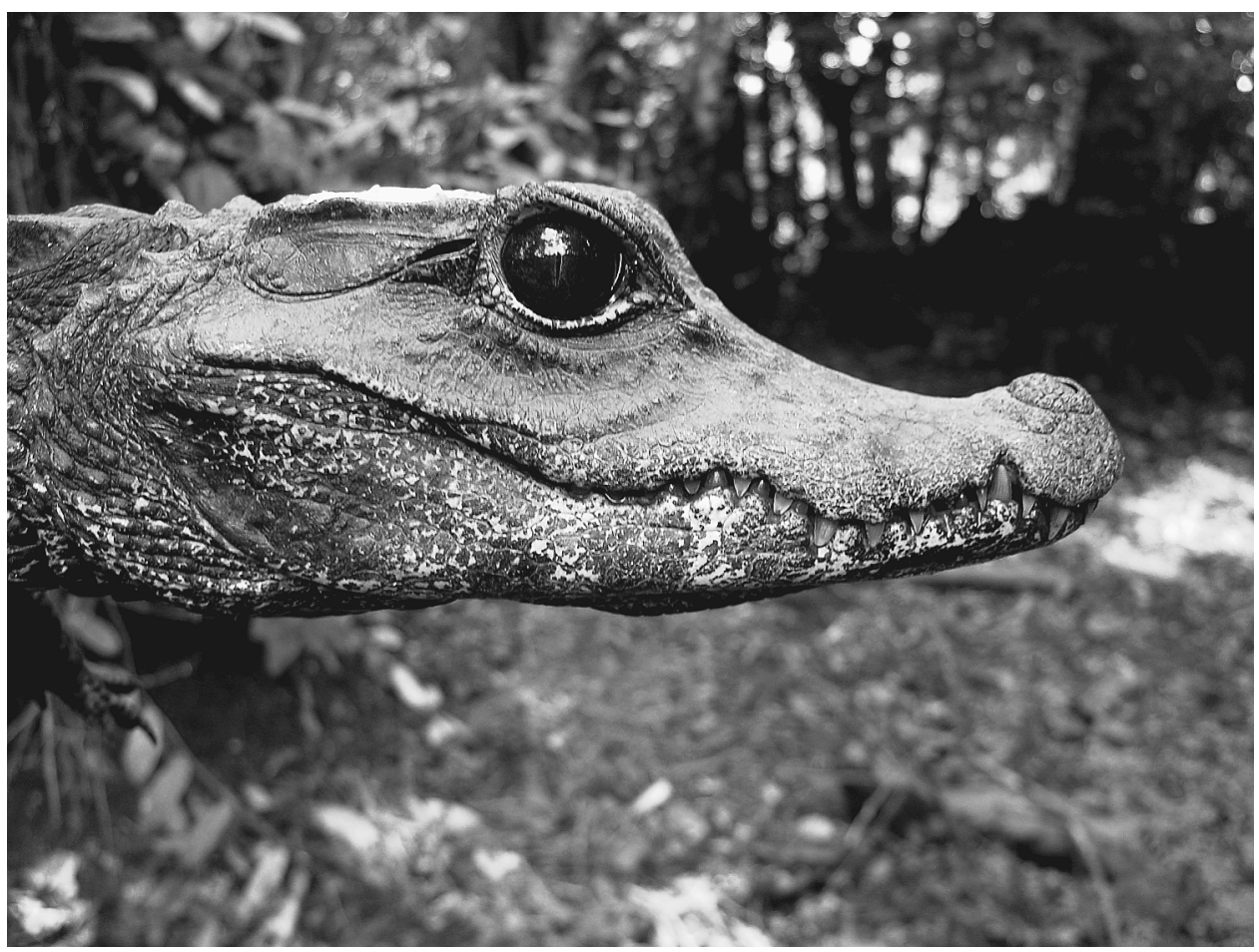

Plate 1. Juvenile male dwarf crocodile (Osteolaemus tetraspis) captured, marked, and measured on 12 July, 2004, in the Republic of Gabon. This individual was observed on the Rabi River near Loango National Park and measured $14.2 \mathrm{~cm}$ head length, $92 \mathrm{~cm}$ total length. Based on the von Bertalanffy growth model presented here, we estimated this crocodile to be approximately eight years old $(90 \% \mathrm{CI}=5.6-17.8)$. Photo credit: M. J. Eaton.

hatchling HL from TL, when combined with information on known-age individuals, allowed predictions of birth size, $S(0)$. Combining these with predictions of asymptotic size $a$ from the recapture data set, we were able to predict values of the parameter $b=1-S(0) / a$.

\section{Predicting age}

Our primary goal was to produce tabulated age predictions based on HL measurements. These predictions are to be used for (hypothetical) future observations, rather than for specific observations in our data set. We use posterior predictive distributions (Gilks et al. 1996, Link and Barker 2010) to properly account for measurement error and inexact knowledge of model parameters.

To establish the relationship between head (HL) and total length (TL), we conducted a regression analysis using data for 515 crocodiles for which we had a complete set of head and body measurements. The relationship,

$$
\mathrm{TL}=-3.795+6.902 \mathrm{HL}
$$

with $R^{2}=0.995$ was used to convert head length to total body length when reporting a predicted age. Because investigators often classify young crocodilians as distinct age classes in mixed-age/stage demographic models (based on higher mortality rates assumed for smaller animals; see Nichols 1987, Tucker 2000), we also use the predictive model and HL to TL conversion to estimate the size of dwarf crocodiles at one and two years of age. By properly assigning field measurements to these age classes, we are able to increase the precision of stage transition estimates and, therefore, improve demographic model projections. As another important state variable boundary used in stage-based demographic models, we predicted the age at which female dwarf crocodiles reach reproductive maturity, based on the minimum size of females observed in the presence of hatchlings (15.0 cm HL; Eaton 2006).

To describe our approach, let matrix $\mathbf{R}$ denote the recapture data, and let vector $\boldsymbol{\theta}=\left(k, a, \sigma_{\mathrm{R}}^{2}\right)$ denote the parameters of growth and error models. Let $\mathbf{S}$ denote the supplementary data matrix and let $\psi=\left(\alpha_{\mathrm{HL}}, \sigma_{\mathrm{HL}}^{2}\right)$ denote the mean and variance of the predictive distribution of $S(0)$. We indicate by $[\boldsymbol{\theta} \mid \mathbf{R}]$ and $[\boldsymbol{\psi} \mid \mathbf{S}]$ the posterior distributions of these parameters, respectively. Because the models for $\mathbf{R}$ and $\mathbf{S}$ share no parameters, and because $\mathbf{R}$ and $\mathbf{S}$ are independent, the joint posterior distribution $[\boldsymbol{\theta}, \boldsymbol{\psi} \mid \mathbf{R}, \mathbf{S}]$ is simply the product $[\boldsymbol{\theta} \mid \mathbf{R}] \times[\boldsymbol{\psi} \mid \mathbf{S}]$; we sample this joint posterior distribution by drawing $\boldsymbol{\theta} \sim[\boldsymbol{\theta} \mid \mathbf{R}]$ and, independently, $\boldsymbol{\psi}$ $\sim[\boldsymbol{\Psi} \mid \mathbf{S}]$.

Suppose that we knew the values of parameter vectors $\boldsymbol{\theta}$ and $\boldsymbol{\psi}$. Given measurement $h$, Bayesian inference for 
TABLE 1. Features of posterior distributions from an integrated growth model to predict the age of African dwarf crocodiles from independent data sets.

\begin{tabular}{cccccc}
\hline \hline & & & \multicolumn{3}{c}{ Posterior percentiles } \\
\cline { 4 - 6 } Parameter & Mean & SD & $2.50 \%$ & $50 \%$ & $97.50 \%$ \\
\hline$a$ & 23.45 & 1.96 & 20.19 & 23.25 & 28.04 \\
$b$ & 0.856 & 0.014 & 0.829 & 0.856 & 0.882 \\
$S[0] \dagger$ & 3.36 & 0.16 & 3.06 & 3.36 & 3.71 \\
$S[1] \dagger$ & 5.36 & 0.94 & 3.63 & 5.32 & 7.39 \\
$S[2] \dagger$ & 7.16 & 1.09 & 5.12 & 7.11 & 9.50 \\
$\alpha_{\mathrm{HL}}$ & 3.36 & 0.10 & 3.19 & 3.36 & 3.57 \\
$\alpha_{\mathrm{TL}}$ & 0.93 & 2.20 & -3.77 & 1.06 & 4.89 \\
$\beta_{\mathrm{HL}}$ & 0.022 & 0.001 & 0.020 & 0.022 & 0.025 \\
$\beta_{\mathrm{TL}}$ & 6.07 & 0.44 & 5.29 & 6.05 & 7.01 \\
$k$ & 0.29 & 0.05 & 0.21 & 0.29 & 0.39 \\
$\lambda$ & 11.16 & 18.70 & 3.17 & 6.55 & 60.64 \\
$\sigma_{\mathrm{HL}}$ & 0.13 & 0.03 & 0.08 & 0.13 & 0.19 \\
$\sigma_{\mathrm{TL}}$ & 1.05 & 0.16 & 0.77 & 1.05 & 1.38 \\
$\sigma_{\mathrm{R}}$ & 0.25 & 0.10 & 0.11 & 0.24 & 0.48 \\
\hline
\end{tabular}

Notes: Data sets included body measurements from recapture observations, single capture events, and a small number of known-age individuals. Abbreviations: HL, head length; TL, total length; $\mathrm{R}$, recapture measurement. Parameters: $a$, asymptotic head length; $b$, a constant relating birth size to asymptotic size; $k$, a growth rate coefficient scaled to 1000 -day intervals; $\lambda$, the ratio of mean to variance for describing individual heterogeneity; $\alpha$ and $\beta$, regression intercept and slope coefficients, respectively, relating expected head length (HL) to animal age, and total length (TL) to head length; $\sigma_{\mathrm{HL}}$ and $\sigma_{\mathrm{TL}}$ are standard deviations of the error terms for true head and total lengths.

$\dagger$ Model-averaged population estimate of head length (HL) at ages 0,1 , and 2 years.

age (AGE) would be based on draws from the posterior distribution $[\mathrm{AGE} \mid \boldsymbol{\theta}, \boldsymbol{\psi}, h] \propto[h \mid \boldsymbol{\theta}, \boldsymbol{\psi}, \mathrm{AGE}][\mathrm{AGE}]$. Instead, we sample values $\boldsymbol{\theta}^{*}$ and $\boldsymbol{\psi}^{*}$ from the posterior distributions $[\boldsymbol{\theta} \mid \mathbf{R}]$ and $[\boldsymbol{\psi} \mid \mathbf{S}]$ and then sample AGE from the distribution $\left[\mathrm{AGE} \mid \boldsymbol{\theta}^{*}, \boldsymbol{\psi}^{*}, h\right]$. In so doing, we are drawing values for $\mathrm{AGE}$ from the posterior predictive distribution

$$
f(\mathrm{AGE} \mid \mathbf{R}, \mathbf{S} ; h)=\int[\mathrm{AGE} \mid \theta, \psi, h][\theta, \psi \mid \mathbf{R}, \mathbf{S}] \partial \theta \partial \psi .
$$

This distribution is distinguished from an ordinary posterior distribution in that $h$ is treated as a hypothetical value: we are predicting AGE for an individual not yet seen, rather than one of the individuals in our data set. The importance of this distinction is that we do not allow this hypothetical observation to inform our inference about parameters $\boldsymbol{\theta}$ and $\psi$.

Joint analysis of $\mathbf{R}$ and $\mathbf{S}$ was conducted using program OpenBUGS, version 3.1.1 (Lunn et al. 2009). Four parallel Markov chains of length 4.5 million were produced; the first 0.5 million values were discarded as burn-in, and every remaining 10th value was recorded, resulting in four chains of length 0.4 million for a total of 1.6 million samples. Run time was roughly $18.5 \mathrm{~h}$ on an i7 $x 980$-based desktop computer. The quality of these Markov chains for evaluating posterior distributions of interest was evaluated by visual inspection of GelmanRubin diagnostics (based on the first 50000 observations in the four thinned chains). Autocorrelations for all nodes died off exponentially, and were negligible at lag 50. Sampling of the posterior predictive distribution $f(\mathrm{AGE} \mid \mathbf{R}, \mathbf{S} ; h)$, while treating $h$ as hypothetical data, is made possible by use of the "cut" command in OpenBUGS (code is available in the Supplement).

\section{Results}

From a total of 619 dwarf crocodiles captured during the study, 46 were recaptured at least once. Annual growth in head length, averaged across all initial capture sizes, was estimated at $1.23 \pm 0.70 \mathrm{~cm} \mathrm{HL} /$ year (mean \pm $\mathrm{SD}$ ), equivalent to $8.49 \mathrm{~cm} \mathrm{TL} / y e a r$, but was size dependent and declined with head length.

A comparison of the von Bertalanffy and logistic growth models using Bayesian multimodel inference overwhelmingly favored the VB to the LG model, with a Bayes factor of $10611(95 \% \mathrm{CI}= \pm 320)$. In the following summary we provide parameter estimates and derived values under the VB model; all point estimates are posterior medians and all interval estimates are $95 \%$ CIs unless otherwise indicated.

The largest head length measurement recorded in the wild $(24.7 \mathrm{~cm})$ fell near the median point prediction for asymptotic head size $(a=23.25)$, and was within the $95 \%$ credible interval $(\mathrm{CI})$ of $(20.2,28.02)$ under the VB model (Table 1). The standard deviation of the measurement error term $\varepsilon_{i j}^{\mathrm{R}}, \sigma_{R}=\sqrt{\sigma_{\mathrm{R}}^{2}}$, was estimated to be $0.25 \mathrm{~cm}(0.11,0.48)$. The point estimate for $b$ (0.856) indicates that the birth size of a dwarf crocodile is about one-seventh its asymptotic size (14.4\%; CI = 11.8-17.2\%). At birth, dwarf crocodiles were predicted to have a head length of $3.36 \mathrm{~cm}(3.06,3.71)$, corresponding to a total length of $19.4 \mathrm{~cm}(\mathrm{CI}=17.3-$ 21.8). By the end of the first year, dwarf crocodiles were predicted to grow to $5.32 \mathrm{~cm} \mathrm{HL}(\mathrm{CI}=3.63-7.39)$ or TL $=32.9 \mathrm{~cm}(\mathrm{CI}=21.3-47.2)$ in the first year, and to reach $7.11 \mathrm{~cm} \mathrm{HL}(\mathrm{CI}=5.11-9.50)$ or $45.3 \mathrm{~cm} \mathrm{TL}(\mathrm{CI}=31.5-$ 61.8) by the end of their second year (Table 1). Based on 


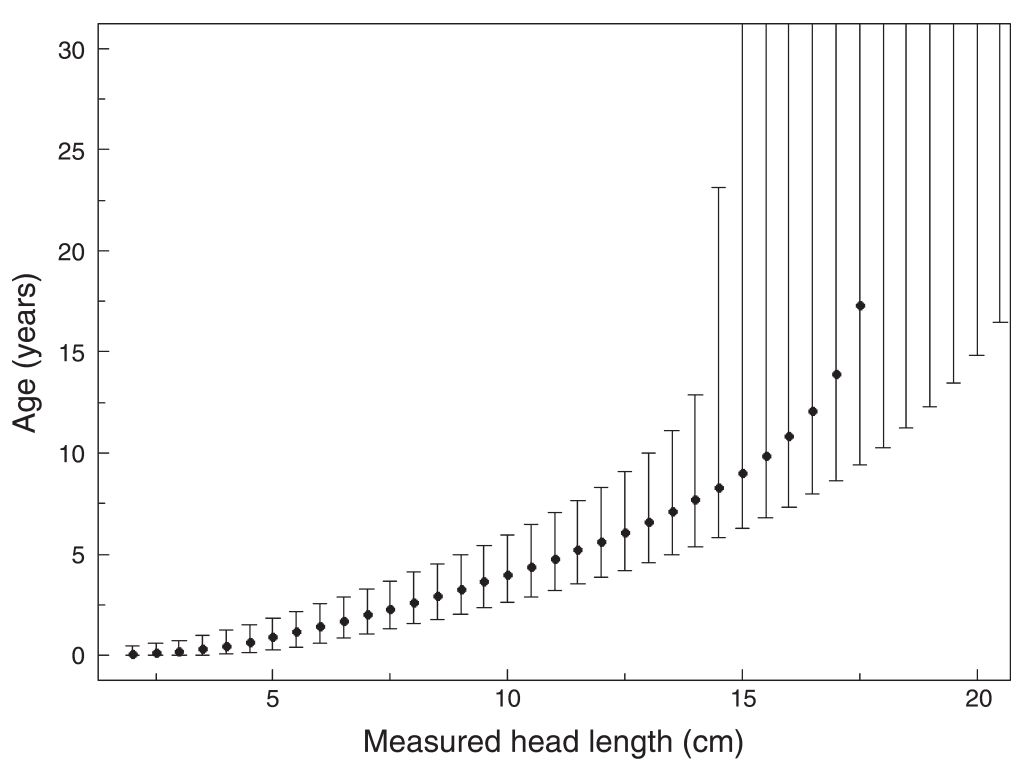

FIG. 3. Posterior median predictions and $90 \%$ prediction intervals for African dwarf crocodile age, given measured head length.

an estimate of $100 \mathrm{~cm}$ TL as the size of reproductive maturity for dwarf crocodiles, the median age of first reproduction was predicted at 9.0 years $(80 \% \mathrm{CI}=6.8-$ 15.7 years; Fig. 3). Asymptotic head length $(23.25 \mathrm{~cm})$ predicted the maximum total length of an adult dwarf crocodile to be $156.7 \mathrm{~cm}(135.6,182.2)$, which very closely approximates our largest measurement recorded in the wild ( $\mathrm{TL}=158.9 \mathrm{~cm})$. Gender-specific estimates of parameters $a$ and $k$ were both slightly higher for male crocodiles, suggesting that males may grow faster and reach a larger asymptotic size than females, but credible intervals overlapped and no significant differences were detected in our data set. Because growth declines with age, the predictive capacity of our model is diminished by the time a crocodile reaches $\sim 15.5 \mathrm{~cm}$ in head length (103 cm TL, median posterior age estimate $=9.9$ years $)$, at which point the upper $90 \%$ percentile becomes quite long (Fig. 3). It is apparent from the model output that the upper quantiles of posterior age predictions are sensitive to priors placed on animal age. This is not surprising, given that the model (justifiably) constrains growth to be asymptotic, and therefore an individual near asymptotic size could be any age beyond the minimum required to approach the asymptote. Because we did not know the life span of dwarf crocodiles in the wild, we felt it was inappropriate to use an informative prior on age. Lower quantiles of posterior age predictions appear to be robust to priors on age.

\section{DiscusSION}

This paper contributes to the study of animal growth, developing an approach that merges information from disparate sources into a common framework to derive predictions of age from observations of animal length. Because growth- and age-specific physiological processes are key determinants of a species' life history strategy, our motivation for developing this modeling structure was to improve the prediction of an individual's age, thereby assisting efforts to conserve and manage poorly known or threatened species through better population models. Reliable methods to determine an individual's age offer several benefits to modeling population dynamics and can aid in species conservation efforts. The ability to derive accurate age predictions by combining disparate data sets collected under routine field studies is valuable for calculating life history traits, including stage transition probabilities, age at first reproduction, and stable age or stage distribution (Cochran and Ellner 1992, Caswell 2001, Spencer 2002). For example, population growth rates in many long-lived species are sensitive to parameters governing the transition from nonbreeding to breeding status (Lebreton and Clobert 1991). Linking observations of reproductive behavior with age predictions to estimate the duration spent in pre-breeding classes (i.e., average age at reproductive maturity), therefore, has significant implications for reducing bias in demographic models, regardless of the model structure (i.e., age- or sizebased). Indeed, deriving measurement-based age estimates from a sampled population, assuming that detection probability is equal across all sizes, allows the investigator the choice between using age- or sizebased methods in modeling that population. Another potential benefit of predicting age from size is the ability to model the distribution of birth dates from captures of young of the year, potentially answering a wide variety of ecological and management-oriented questions related to timing of parturition (Mazzotti et al. 1986, Platt and Thorbjarnarson 2000).

In the example presented here, we integrated three modest data sets to predict the age of a hypothetical African dwarf crocodile of a given length. Data sets 
included incremental growth records from a markrecapture study, measurements from a small sample of young individuals, and a few observations of known-age animals used to estimate the final growth parameter, birth size. The von Bertalanffy growth model, commonly used for describing the growth of reptiles, was determined to be a substantially better predictor of age than the logistic model for our particular data. By combining these data under a common framework, relatively narrow credible intervals for age were estimated for animals up to $\sim 66 \%$ of asymptotic size (15.5 $\mathrm{cm}$ HL or $103 \mathrm{~cm} \mathrm{TL})$. Beyond this size, growth rate declined and size became a poor predictor of age. Under any model in which size asymptotes with age, size-based age prediction is inevitably difficult for animals captured when nearly fully grown. Although age estimates for large dwarf crocodiles were imprecise, lower credible intervals still provided useful information on minimum age. For example, an animal nearing asymptotic size (i.e., $21 \mathrm{~cm}$ HL, $141 \mathrm{~cm}$ TL) was estimated with $90 \%$ probability to be a minimum of 16.5 years old. Our estimate for age at reproductive size for dwarf crocodiles ( $80 \% \mathrm{CI}=6.8-15.7$ years) was very similar to the range observed for mature females in captive populations (513 years; Tryon 1980, Schmidt 2007).

The species that we selected for demonstrating the model represents a group of animals for which independent age estimation is not possible, but the general statistical approach presented is applicable to a variety of incremental growth data for any species that can be fit to a growth model. Capture-recapture studies have become standard practice in animal ecology, offering valuable sources of information that are not always exploited to their full benefit. The opportunity to integrate additional sources of information, many forms of which are collected in standard field studies, into a common framework is the primary contribution of our model and should be appealing to many investigators. Such an approach is not limited to the analysis of growth, but can be applied to other areas of ecology including parameter estimation for population demographic modeling (i.e., Cave et al. 2010) and, in general, the merging of process and observation models (Harwood and Stokes 2003). The strength of our approach lies in the flexibility of the model structure, which can assimilate independent data sets into a single modeling framework to maximize available information while also permitting missing data and accommodating multimodel inference. With small data sets, such as ours, the inclusion of individual heterogeneity of modeled growth parameters through random effects terms can be problematic. Our data set, for example, contained only five observations of animals captured more than twice, making it difficult to directly estimate individual heterogeneity in parameters such as asymptotic length (a) or growth rate $(k)$. Such limitations for estimating individual variability in multiple growth parameters are well recognized (Hart and Chute 2009). We used a
Gamma process model to incorporate individual variability in growth patterns, allowing us to model the expected population mean head length while treating true individual head size as a latent variable. The Gamma process is an appealing and convenient model for analysis of growth based on capture-recapture data, in that it specifies a nondecreasing, continuous-time Markov process with independent increments.

The growth model that we have developed applies advances in Bayesian and hierarchical modeling to combine independent data sets into an integrated framework for inference on the age of animals based only on body size. The formulation of this model and the analysis of the crocodile data sets provided a few valuable insights that we include as recommendations to other investigators. First, because growth models depend heavily on multiple recaptures to estimate asymptotic size and, in our case, predict age, carefully considering the study's sampling design to maximize the probability of encountering individuals on multiple occasions will improve model inference. Second, with regard to higher upper bounds on credible intervals for animals approaching asymptotic size, one possible solution to increase the precision for predicting the age of older animals is to incorporate informative priors on maximum age. Such data could come from other studies of the same species, from related species, or from captive records. As a final caveat, we caution against applying estimated parameter values and age predictions to populations outside the direct geographic region of inference, because growth is often environmentally mediated and geographically variable.

\section{ACKNOWLEDGMENTS}

We thank Jim Nichols, Andy Royle, Ellen Bean, Mike Runge, and two anonymous reviewers for insightful comments on earlier drafts of this manuscript. M. J. Eaton thanks Brett Melbourne for the initial conceptualization of a model for age predictions, assistants at Operation Loango, Gabon, for their effort in the field, and the Wildlife Conservation Society and the Société pour la Conservation et le Development for logistical and financial support. We dedicate this manuscript to the memory of our colleague, John Thorbjarnarson (1957-2010). Use of trade, product, or firm names does not imply endorsement by the U.S. Government.

\section{Literature Cited}

Alexander, M. M. 1958. The place of aging in wildlife management. American Scientist 46:123-137.

Applebaum, D. 2009. Lévy processes and stochastic calculus. Cambridge University Press, Cambridge, UK.

Avens, L., J. C. Taylor, L. R. Goshe, T. T. Jones, and M. Hastings. 2009. Use of skeletochronological analysis to estimate the age of leatherback sea turtles Dermochelys coriacea in the western North Atlantic. Endangered Species Research 8:165-177.

Bacon-Shone, J. 1988. Bayesian methods for growth curves with capture-recapture. Journal of the Royal Statistical Society, Series D (The Statistician) 37:307-312.

Bjorndal, K. A., A. B. Bolten, R. A. Bennett, E. R. Jacobson, T. J. Wronski, J. J. Valeski, and P. J. Eliazar. 1998. Age and growth in sea turtles: limitations of skeletochronology for demographic studies. Copeia 1998:23-30. 
Bjorndal, K. A., A. B. Bolten, T. Dellinger, C. Delgado, and H. R. Martins. 2003. Compensatory growth in oceanic loggerhead sea turtles: response to a stochastic environment. Ecology 84:1237-1249.

Cailliet, G. M., H. F. Mollet, G. G. Pittenger, D. Bedford, and L. J. Natanson. 1992. Growth and demography of the Pacific angle shark (Squatina californica), based upon tag returns off California. Marine and Freshwater Research 43:1313-1330.

Caswell, H. 2001. Matrix population models: construction, analysis, and interpretation. Second edition. Sinauer Associates, Sunderland, Massachusetts, USA.

Cave, V. M., R. King, and S. N. Freeman. 2010. An integrated population model from constant effort bird-ringing data. Journal of Agricultural, Biological, and Environmental Statistics 15:119-137.

Cheng, Y. W., and A. Y. C. Kuk. 2002. Determination of the unknown age at first capture of western rock lobsters (Panulims cygnus) by random effects model. Biometrics 58:459-462.

Clark, J. S. 2005. Why environmental scientists are becoming Bayesians. Ecology Letters 8:2-14.

Clark, J. S., G. A. Ferraz, N. Oguge, H. Hays, and J. DiCostanzo. 2005. Hierarchical Bayes for structured, variable populations: from recapture data to life-history prediction. Ecology 86:2232-2244.

Clarke, C. M. H., R. M. Dzieciolowski, D. Batcheler, and C. M. Frampton. 1992. A comparison of tooth eruption and wear and dental cementum techniques in age determination of New Zealand feral pigs. Wildlife Research 19:769-777.

Clawson, R. G., and M. K. Causey. 1995. Dental casts for white-tailed deer age estimation. Wildlife Society Bulletin 23:92-94.

Cochran, M. E., and S. P. Ellner. 1992. Simple methods for calculating age-based life history parameters for stagestructured populations. Ecological Monographs 62:345-364.

Cole, L. 1954. The population consequences of life history phenomena. Quarterly Review of Biology 29:103-137.

Eaton, M. J. 2006. Ecology, conservation and management of the Central African dwarf crocodile (Osteolaemus tetraspis), a progress report. Pages 84-95 in Crocodiles: Proceedings of the 18th Working Meeting of the IUCN-SSC Crocodile Specialist Group. IUCN, Gland, Switzerland.

Eaton, M. J., A. P. Martin, J. Thorbjarnarson, and G. Amato. 2009. Species-level diversification of African dwarf crocodiles (Genus Osteolaemus): a geographic and phylogenetic perspective. Molecular Phylogenetics and Evolution 50:496-506.

Fabens, A. J. 1965. Properties and fitting of von Bertalanffy growth curve. Growth 29:265-289.

Franken, R. J., and D. S. Hik. 2004. Interannual variation in timing of parturition and growth of collared pikas (Ochotona collaris) in the southwest Yukon. Integrative and Comparative Biology 44:186-193.

Gilks, W. R., S. Richardson, and D. J. Spiegelhalter. 1996. Markov chain Monte Carlo in practice. Chapman and Hall, London, UK.

Gross, K., B. A. Craig, and W. D. Hutchison. 2002. Bayesian estimation of a demographic matrix model from stagefrequency data. Ecology 83:3285-3298.

Hart, D. R., and A. S. Chute. 2009. Estimating von Bertalanffy growth parameters from growth increment data using a linear mixed-effects model, with an application to the sea scallop Placopecten magellanicus. ICES Journal of Marine Science 66:2165-2175.

Harwood, J., and K. Stokes. 2003. Coping with uncertainty in ecological advice: lessons from fisheries. Trends in Ecology and Evolution 18:617-622.

Haussmann, M. F., and C. M. Vleck. 2002. Telomere length provides a new technique for aging animals. Oecologia 130:325-328.

He, J. X., and J. R. Bence. 2007. Modeling annual growth variation using a hierarchical Bayesian approach and the von
Bertalanffy growth function, with application to lake trout in southern Lake Huron. Transactions of the American Fisheries Society 136:318-330.

Hearn, B. J., and W. E. Mercer. 1988. Eye-lens weight as an indicator of age in Newfoundland arctic hares. Wildlife Society Bulletin 16:426-429.

Helser, T. E., and H.-L. Lai. 2004. A Bayesian hierarchical meta-analysis of fish growth: with an example for North American largemouth bass, Micropterus salmoides. Ecological Modelling 178:399-416.

Helser, T. E., I. J. Stewart, and H.-L. Lai. 2007. A Bayesian hierarchical meta-analysis of growth for the genus Sebastes in the eastern Pacific Ocean. Canadian Journal of Fisheries and Aquatic Sciences 64:470-485.

Hutton, J. M. 1987a. Growth and feeding ecology of the Nile crocodile Crocodylus niloticus at Ngezi, Zimbabwe. Journal of Animal Ecology 56:25-38.

Hutton, J. M. 1987b. Techniques for ageing wild crocodilians. Pages 211-216 in G. J. W. Webb, S. C. Manolis, and P. J. Whitehead, editors. Wildlife management: crocodiles and alligators. Surrey Beatty, Chipping Norton, New South Wales, Australia.

Ims, R. A. 1990. The ecology and evolution of reproductive synchrony. Trends in Ecology and Evolution 5:135-140.

James, I. R. 1991. Estimation of von Bertalanffy growth curve parameters from recapture data. Biometrics 47:1519-1530.

Kimura, D. K. 1980. Likelihood methods for the von Bertalanffy growth curve. Fisheries Bulletin 77:765-775.

Kimura, D. K. 2008. Extending the von Bertalanffy growth model using explanatory variables. Canadian Journal of Fisheries and Aquatic Sciences 65:1879-1891.

Kofron, C. P. 1992. Status and habitats of the three African crocodiles in Liberia. Journal of Tropical Ecology 8:265-273.

Kohn, L. A. P., P. Olson, and J. M. Cheverud. 1997. Age of epiphyseal closure in tamarins and marmosets. American Journal of Primatology 41:129-139.

Laundré, J. W., and L. Hernández. 2002. Growth curve models and age estimation of young cougars in the Northern Great Basin. Journal of Wildlife Management 66:849-858.

Leberg, P. L., I. L. Brisbin, M. H. Smith, and G. C. White. 1989. Factors affecting the analysis of growth-patterns of large mammals. Journal of Mammalogy 70:275-283.

Lebreton, J.-D., and J. Clobert. 1991. Bird population dynamics, management and conservation: the role of mathematical modelling. Pages $105-125$ in C. M. Perrins, J.-D. Lebreton, and G. J. M. Hirons, editors. Bird population studies. Oxford University Press, Oxford, UK.

Leopold, A. 1933. Game management. Charles Scribner's, New York, New York, USA.

Link, W. A., and R. J. Barker. 2010. Bayesian inference: with ecological applications. Elsevier/Academic Press, Amsterdam, The Netherlands.

Liu, Y., L.-R. Jin, W. Metzner, and J. Feng. 2009. Postnatal growth and age estimation in big-footed myotis, Myotis macrodactylus. Acta Chiropterologica 11:105-111.

Lunn, D. J., D. Spiegelhalter, A. Thomas, and N. Best. 2009. The BUGS project: evolution, critique and future directions. Statistics in Medicine 28:3049-3067.

Mazzotti, F., B. Bohnsack, M. P. Mcmahon, and J. R. Wilcox. 1986. Field and laboratory observations on the effects of high temperature and salinity on hatchling Crocodylus acutus. Herpetologica 42:191-196.

Moore, J. E., and A. J. Read. 2008. A Bayesian uncertainty analysis of cetacean demography and bycatch mortality using age-at-death data. Ecological Applications 18:1914-1931.

Moore, N. P., J. P. Cahill, P. F. Kelly, and T. J. Hayden. 1995. An assessment of five methods of age determination in an enclosed population of fallow deer (Dama dama). Biology and Environment 95B:27-34.

Nichols, J. D. 1987. Population models and crocodile management. Pages 177-187 in G. J. W. Webb, S. C. 
Manolis, and P. J. Whitehead, editors. Wildlife management: crocodiles and alligators. Surrey Beatty, Chipping Norton, New South Wales, Australia.

Pilling, G. M., G. P. Kirkwood, and S. G. Walker. 2002. An improved method for estimating individual growth variability in fish, and the correlation between von Bertalanffy growth parameters. Canadian Journal of Fisheries and Aquatic Sciences 59:424-432.

Platt, S. G., and J. Thorbjarnarson. 2000. Nesting ecology of the American crocodile in the coastal zone of Belize. Copeia 2000:869-873.

Rachlow, J. L., and R. T. Bowyer. 1991. Interannual variation in timing and synchrony of parturition in Dall sheep. Journal of Mammalogy 72:487-492.

Rasch, R., A. D. Tucker, L. Daddow, and E. Wentrup-Byrne. 2000. Electron microprobe investigations of growth marks in crocodile osteoderms. Pages $144-155$ in G. C. Grigg, F. Seebacher, and C. E. Franklin, editors. Crocodilian biology and evolution. Surrey Beatty, Chipping Norton, New South Wales, Australia.

Reilly, J. 2002. Growth in the Sumatran elephant (Elephas maximus sumatranus) and age estimation based on dung diameter. Journal of Zoology, London 258:205-213.

Riley, J., and F. W. Huchzermeyer. 1999. African dwarf crocodiles in the Likouala swamp forests of the Congo Basin: habitat, density, and nesting. Copeia 1999:313-320.

Russo, T., P. Baldi, A. Parisi, G. Magnifico, S. Mariani, and S. Cataudella. 2009. Lévy processes and stochastic von Bertalanffy models of growth, with application to fish population analysis. Journal of Theoretical Biology 258:521-529.

Schmidt, F. 2007. European studbook for the African dwarf crocodile (Osteolaemus tetraspis). Zoo Leipzig, Leipzig, Germany.

Schoener, A., and T. W. Schoener. 1978. Estimating and interpreting body-size growth in some Anolis lizards. Copeia 1978:390-405.

Schwarz, L. K., and M. C. Runge. 2009. Hierarchical Bayesian analysis to incorporate age uncertainty in growth curve analysis and estimates of age from length: Florida manatee (Trichechus manatus) carcasses. Canadian Journal of Fisheries and Aquatic Sciences 66:1775-1789.

Spencer, R.-J. 2002. Growth patterns of two widely distributed freshwater turtles and a comparison of common methods to estimate age. Australian Journal of Zoology 50:477-490.
Stander, P. E. 1997. Field age determination of leopards by tooth wear. African Journal of Ecology 35:156-161.

Stearns, S. C. 1992. The evolution of life histories. Oxford University Press, Oxford, UK.

Thorbjarnarson, J. B., and M. J. Eaton. 2004. Preliminary examination of crocodile bushmeat issues in the Republic of Congo and Gabon. Pages 236-247 in Crocodiles: Proceedings of the 17th Working Meeting of the IUCN-SSC Crocodile Specialist Group. IUCN, Gland, Switzerland.

Tovar-Avila, J., V. S. Troynikov, T. I. Walker, and R. W. Day. 2009. Use of stochastic models to estimate the growth of the Port Jackson shark, Heterodontus portusjacksoni, off eastern Victoria, Australia. Fisheries Research 95:230-235.

Tryon, B. W. 1980. Observations on reproduction in the west African dwarf crocodile, with a description of parental behavior. Society for the Study of Amphibians and Reptiles, Contributions to Herpetology 1:167-185.

Tucker, A. D. 1997. Validation of skeletochronology to determine age of freshwater crocodiles (Crocodylus johnstoni). Marine and Freshwater Research 48:343-351.

Tucker, A. D. 2000. Sensitivity analysis of stage-structured demographic models for freshwater crocodiles. Pages 349363 in G. C. Grigg, F. Seebacher, and C. E. Franklin, editors. Crocodilian biology and evolution. Surrey Beatty, Chipping Norton, new South Wales, Australia.

Wade, P. R. 2000. Bayesian methods in conservation biology. Conservation Biology 14:1308-1316.

Waitkuwait, W. E. 1989. Present knowledge on the West African slender-snouted crocodile, Crocodylus cataphractus Cuvier 1824 and the West African dwarf crocodile Osteolaemus tetraspis, Cope 1861. Pages 260-275 in P. M. Hall and R. J. Bryant, editors. Crocodiles: their ecology, management, and conservation. IUCN, Gland, Switzerland.

Webb, G. J. W., R. Buckworth, and C. Maniolis. 1983. Crocodylus johnstoni in the McKinlay River area, NT. Growth, movement and the population age structure. Australian Wildlife Research 10:383-401.

Williams, B. K., J. D. Nichols, and M. J. Conroy. 2002. Analysis and management of animal populations: modeling, estimation, and decision making. Academic Press, San Diego, California, USA.

Wilson, V. J., J. L. Schmidt, and J. Hanks. 1984. Age determination and body growth of the common duiker Sylvicapra grimmia (Mammalia). Journal of Zoology 202:283-297.

\section{APPENDIX A}

Recapture data for 46 African dwarf crocodiles, including date of original and subsequent captures, head length at each capture, and time in days between captures (Ecological Archives A021-110-A1).

\section{APPENDIX B}

Head and total length of 51 young dwarf crocodiles used to estimate birth size parameter for Logistic and von Bertalanffy growth models (Ecological Archives A021-110-A2).

\section{SUPPLEMENT}

OpenBUGS code for modeling recapture data and measurements of young crocodiles of known and unknown ages (Ecological Archives A021-110-S1). 